\title{
Residual esophageal necrosis after radical esophagectomy for esophagogastric cancer: A case report
}

\author{
SHUNSUKE TABE $^{1,2}$, ISAMU HOSHINO ${ }^{1}$, NOBUHIRO TAKIGUCHI ${ }^{1}$, ATSUSHI IKEDA ${ }^{1}$, HIROAKI SODA ${ }^{1}$, \\ TORU TONOOKA ${ }^{1}$, HISASHI GUNJI ${ }^{1}$, YOSHIHIRO NABEYA ${ }^{1}$ and MASAYUKI OTSUKA ${ }^{1,2}$ \\ ${ }^{1}$ Department of Gastroenterological Surgery, Chiba Cancer Center, Chiba 260-8717; \\ ${ }^{2}$ Department of General Surgery, Chiba University Graduate School of Medicine, Chiba 260-8677, Japan
}

Received October 1, 2019; Accepted January 31, 2020

DOI: $10.3892 / \operatorname{mco} .2020 .1997$

\begin{abstract}
A 66-year-old man was diagnosed with advanced esophagogastric junction cancer and referred to our institution (Department of Gastroenterological Surgery, Chiba Cancer Center) for treatment. Computed tomography imaging confirmed the presence of a tumor, extending from the lower thoracic esophageal to the esophagogastric junction, with swelling of the upper mediastinal lymph nodes. Based on the criteria of the International Union against Cancer Committee (UICC, 8th Edition), the staging was confirmed as follows: 101R, 107 and 106 pre. Based on these findings, a clinical diagnosis of EGJ cancer was made, with a UICC8th classification of cT3N1M0 c-stage-III. Preoperative chemotherapy was performed, with tumor shrinkage obtained after three courses of chemotherapy (using S-1 plus oxaliplatin). Subsequently, esophagectomy with three-field lymph node dissection and gastric tube reconstruction, via the intrathoracic route, was performed. On postoperative day 2 , the patient developed an idiopathic pneumothorax, with brown-green drainage from the chest tube. A repeat thoracotomy was performed, confirming the presence of brown-green pleural fluid and necrosis of esophageal tissue. The area of necrosis was situated $4 \mathrm{~cm}$ on the oral side of the anastomosis, with greater necrosis of the right than left side. There was no evidence of necrosis of the gastric tube. The necrotic residual esophagus was excised and reconstructed, as an external fistula on the left side of the neck. On day 38 ,
\end{abstract}

Correspondence to: Dr Isamu Hoshino, Department of Gastroenterological Surgery, Chiba Cancer Center, 666-2 Nitonacho, Chuo-ku, Chiba 260-8717, Japan

E-mail: ihoshino@chiba-cc.jp

Abbreviations: EGJ, esophagogastric junction; UICC, International Union against Cancer Committee; PET-CT, positron-emission tomography computed tomography; CT, computed tomography; NAC, neoadjuvant chemotherapy; POD, postoperative day

Key words: residual esophageal necrosis, esophagectomy, esophagogastric cancer after the second surgery, reconstruction of the esophageal conduit and gastric tube, via the jejunum, was performed. At 7 months after discharge, the patient was symptom free, with no evidence of cancer recurrence.

\section{Introduction}

In recent years, the frequency of esophagogastric junction cancer (EGJ) has been rapidly increasing in Europe and the United States. According to Comprehensive registry of esophageal cancer in japan 2010, the incidence of EGJ cancer is also increasing (1). The EGJ cancers are classified by Rudiger Siewert et al (2) and different types require different treatment strategies, therefore there is no established consensus on the optimal treatment.

In Europe neoadjuvant-chemoradiotherapy (neo-CRT) is the standard treatment for EGJ cancer based on the CROSS trial (3), but it is concerned that the effect of CRT is lower in adenocarcinoma than in squamous cell carcinoma.

Radical esophagectomy is considered as the standard treatment for patients with esophagogastric junction (EGJ) cancer with extensive invasion of the esophagus and/or suspected metastasis to the mediastinal area. With recent advances in surgical technique and perioperative management, the rate of perioperative mortality for radical esophagectomy has been reported to be $<5 \%$ (4). The latest study which based on the Japanese national clinical database reported that the overall 30 -day mortality rate for open esophagectomy was $0.9 \%$, and total surgery related mortality rate was $2.4 \%$ (5). Furthermore, it is suggested that minimally invasive esophagectomy (MIE) may be performed and may contribute to reduction of complications. However, complications during anastomosis which causes mediastinitis and empyema thoracis can led to septic shock may be severe and, thus, caution is required. Specifically, necrosis of the esophageal anastmosis reconstruction conduit after esophagectomy is a complication that carries a risk of mortality of up to $90 \%$ (6). The reconstructed conduit necrosis is rare and is only reported in $<2 \%$ of primary resections with reconstruction (6). Herein, we describe the case of a patient with necrosis in the residual esophagus, rather than in the reconstructed conduit. We include our treatment and the clinical outcome. 


\section{Case report}

A 66-year-old man presented to Asai Hospital (Chiba, Japan) with epigastric discomfort and symptoms of gastrointestinal obstruction. Gastrointestinal endoscopy revealed a tumor at the EGJ, which was confirmed as an adenocarcinoma on biopsy. Contrast-enhanced computed tomography (CT) was performed at our institution, which revealed a tumor extending from the lower thoracic esophagus to the EGJ, with associated swelling of the upper mediastinal lymph nodes. Based on the criteria of the International Union against Cancer Committee (UICC, 8th Edition), the clinical staging was as follows: 101R, 107, and 106 pre. There was little suspicion of lymph node metastasis owing to the small amount of accumulation observed on positron-emission tomography (PET) CT (PET-CT) imaging (Fig. 1A and B). Laboratory tests revealed abnormal values of creatinine level $(1.14 \mathrm{mg} / \mathrm{dl}$; reference range $0.65-1.07)$, with all other levels being within normal range. Tumor marker levels were as follows: Carcinoembryonic antigen, $4.7 \mathrm{ng} / \mathrm{dl}$ (upper reference limit, $5.0 \mathrm{ng} / \mathrm{dl}$ ); carbohydrate antigen 19-9, $6.9 \mathrm{IU} / \mathrm{ml}$ (upper reference limit, $37.0 \mathrm{IU} / \mathrm{ml}$ ); and cancer antigen 125, $14.5 \mathrm{IU} / \mathrm{ml}$ (upper reference limit, $35.0 \mathrm{IU} / \mathrm{ml}$ ). Based on these findings, we made a clinical diagnosis of EGJ cancer, with a UICC8th classification of cT3N1M0 c-stage-III.

In accordance with this diagnosis, preoperative S-1 neoadjuvant chemotherapy (NAC) was implemented (oral fluoropyrimidine, containing tegafur, gimeracil, and oteracil potassium) in combination with oxaliplatin. After 3 courses of NAC, the size of the tumor was reduced, but without a clinical change in lymphadenopathy, with still little accumulation of PET-CT scans (Fig. 2). Based on these findings, we proceeded with esophagectomy, with three-field lymph node dissection and gastric tube reconstruction, via an intrathoracic route. Surgery was performed 4 weeks after the chemotherapy.

We first proceeded with cervical surgery. The right reflex nerve was identified during right cervical lymph node dissection (101R), with observation of an enlarged lymph node. Accordingly, we proceeded with bilateral dissection of the lymph nodes dissection, but with no observable evidence of lymphadenopathy on the left side. The abdominal component of the surgery was performed as per usual methods, with the stomach used to reconstruct the esophageal conduit. Following upper, middle and lower mediastinal lymphadenectomy, we proceeded with reconstruction of the gastric tube, with dissection of the right bronchial artery and azygos vein. The surgical time was $318 \mathrm{~min}$, with a volume of blood loss of $210 \mathrm{ml}$.

An idiopathic pneumothorax developed on postoperative day (POD) 2, with a brown-green drainage from the chest tube. The pneumothorax was confirmed on CT imaging, but with no evidence of abnormality around the anastomosis. As the pneumothorax did not improve with conservative treatment, with persistent green drainage in the thoracic tube, we proceeded with surgical management of the pneumothorax and observation of the anastomosis (Fig. 3). On repeat thoracotomy, the continued presence of brown-green pleural fluid was confirmed, and necrosis of the esophagus was observed, after release of the belaq attached to the anastomosis. The area of necrosis was localized $4 \mathrm{~cm}$ on the oral side of the anastomosis, with necrotic damage being more prominent on the right than left side, but with no signs of necrosis in the gastric tube (Fig. 4).

We proceeded with excision of the residual necrotic esophagus, followed with reconstruction of an external fistula on left side of neck. The residual gastric tube was positioned under the skin, through an antethoracic route. The pneumothorax was detected in the S1 area and closed using staples.

Post-surgery, the patient did not develop further complications and was discharged on POD 17 after the second surgery. On POD 38 (after the second surgery), we proceeded with reconstruction to connect the residual esophagus and gastric tube, through the jejunum. Pathological findings of the resected specimen confirmed residual adenocarcinoma at the esophagogastric junction, with the following tumor components identified: Tub1 $>$ tub2 and $>$ por 2 . The resected margin was negative. The final pathological diagnosis was ypT1aN0(0/66)M0, ypStageIA (UICC8th). Owing to the impairment in the local circulation, necrotizing tissue was observed in the entire layer of the residual esophagus, but not in the layer of the conduit (Fig. 5A and B). At 7 months post-discharge, the patient was alive, with no evidence of cancer recurrence.

\section{Discussion}

It is very rare for residual esophageal necrosis to occur after esophagectomy. Although conduit necrosis after esophagectomy has previously been reported, we identified only one report regarding necrosis of the esophagus (7). Blood flow to the conduit after esophagectomy is primarily dependent on the mucosal capillary network (8). The nature of this capillary network, however, has not been clearly determined. Takemura et al (7) argued that the capillary network developed in the submucosal layer would sustain the residual esophagus, despite inclusion of the main artery along with lymph node dissection.

The following factors can contribute to necrosis of the esophageal conduit $(6,9)$ : Diabetes, hypertension, and peripheral vascular disease. However, smoking, preoperative chemotherapy, and bodyweight were not identified as contributing factors to conduit necrosis (9). The mortality rate associated with necrosis of the esophageal conduit can be as high as $90 \%$ (6). We note that the patient in the case study reported by Takemura et al (7), had a history of myocardial infarction and vascular disease-factors that were not present in our case. The factors that cause necrosis remain unknown in this patient.

Acute ischemic necrosis of the esophagus is possible, as occurs in bowel disease, although the etiology of this acute necrosis is different in the esophagus and bowel. Necrosis of the esophagus (also known as black esophagus) is very rare, with a prevalence of $0.2 \%$ in autopsy data (10). The etiology of black esophagus is associated with risk factors for atherosclerosis (such as hypertension, diabetes, and ageing) or diabetic ketoacidosis, as well as multiple organ dysfunction and sepsis (11). However, none of these factors were identified in our case. As such, it is likely that performance of the surgical anastomosis itself was the cause of esophageal necrosis in our case. 

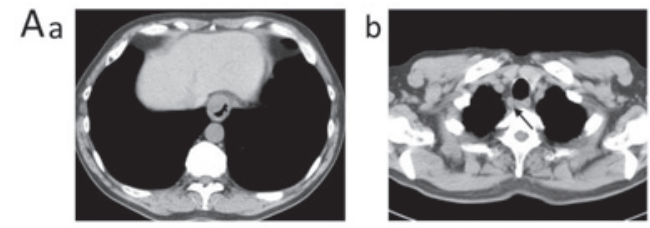

B

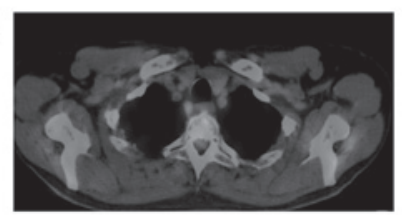

Figure 1. Images before chemotherapy. (Aa) Computed tomography revealed a tumor extending from the lower thoracic esophagus to the esophagogastric junction. (Ab) Swelling of the upper mediastinal lymph nodes. (B) As there was little evidence of accumulation in the lymph nodes, the likelihood of lymph node metastasis was deemed to be low.

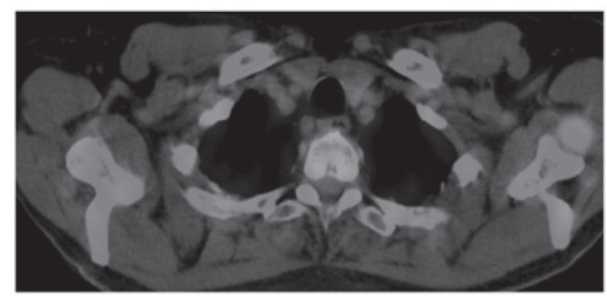

Figure 2. After 3 courses of NAC, there was little accumulation in the lymph nodes. NAC, neoadjuvant chemotherapy.
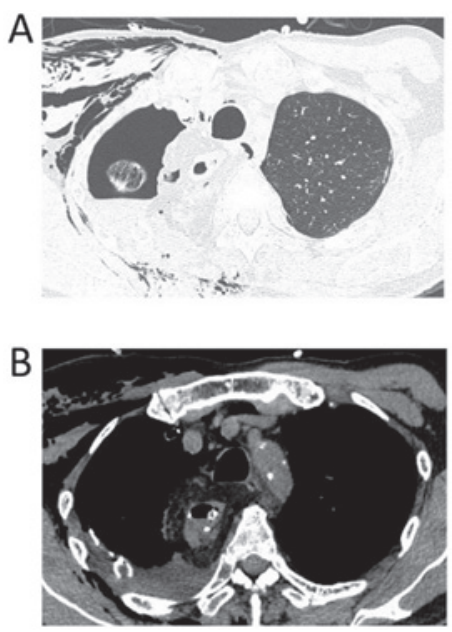

Figure 3. Images after the radical esophagectomy. (A) Computed tomog raphy revealed a pneumothorax in right side. (B) There was no observable abnormality around the anastomosis.

The enlarged right lymph node (101R) was strongly adherent to the wall of the esophagus. Therefore, the possibility of direct invasion of the cancer into the esophageal wall could not be ruled out. During dissection, the muscle layer of the esophagus was excised. Once the 101 lymph nodes were dissected, the cervical esophagus was excised from the surrounding area and released, which differed from traditional cervical dissection and esophagectomy. We do note that this caused a disruption of the local blood flow to the right side of the esophageal wall. Takemura et al (7) reported that the

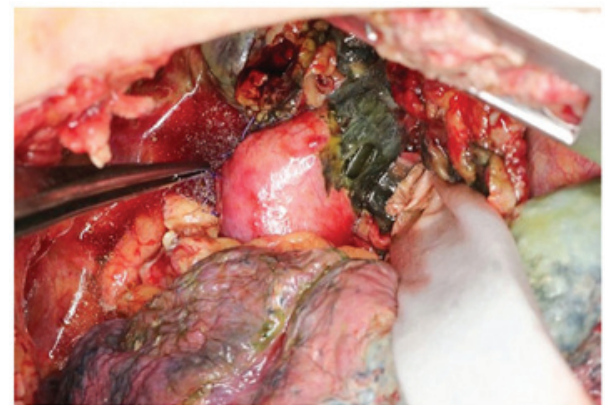

Figure 4. Necrosis of the esophagus, situated $4 \mathrm{~cm}$ of the oral side of the anastomosis. Greater damage was observed on the right compared with the left esophagus, with no evidence of necrosis of the gastric tube.

$\mathrm{Aa}$
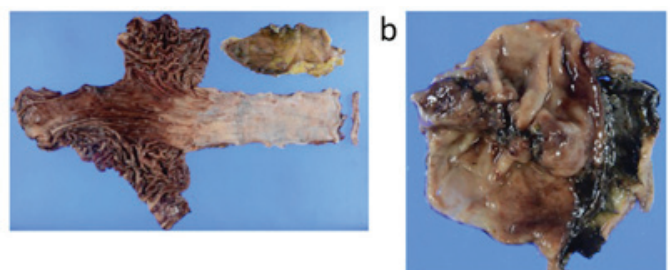

B

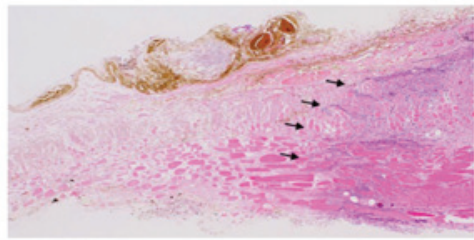

Figure 5. Pathological findings. (Aa) Resected specimen of the adenocarcinoma at the esophagogastric junction. (Ab) Resected specimen after second operation. Necrosis of residual esophagus was observed in right side. (B) Necrotizing tissue was observed through the entire layer of the residual esophagus (black arrow), with no evidence of necrosis in layers of the reconstructed conduit.

length of the residual esophagus might be one of the causes of necrosis, but the length of residual esophagus in our case was normal. Although it remains unclear what caused necrosis of the residual esophagus in our case, it is possible that the wide anastomosis, which extended into the cervical area, might be an important factor to consider.

Residual esophageal necrosis after esophagectomy is very rare and the possible causative factors remain to be fully clarified. Based on our experience, extensive dissection of the cervical esophagus area might be a contributing factor, due to a deterioration of the local blood flow.

\section{Acknowledgements}

Not applicable.

\section{Funding}

No funding was received.

\section{Availability of data and materials}

All data generated or analyzed during this study are included in this published article. 


\section{Authors' contributions}

ST and IH designed the study and wrote the initial draft of the manuscript. IH, NT, AI, HS, TT, HG, YN and MO contributed to design and assisted in the preparation of the manuscript. All other authors have contributed to data collection and interpretation, and critically reviewed the manuscript. All authors approved the final version of the manuscript, and agree to be accountable for all aspects of the work in ensuring that questions related to the accuracy or integrity of any part of the work are appropriately investigated and resolved.

\section{Ethics approval and consent to participate}

This study was approved by the Institutional Ethics Review Board of the Chiba Cancer Center (H29-262) (Chiba, Japan).

\section{Patient consent for publication}

Written informed consent was obtained from the patient for publication of this case report and any accompanying images.

\section{Competing interests}

The authors declare that they have no competing interests.

\section{References}

1. Tachimori Y, Ozawa S, Numasaki H, Ishihara R, Matubara H, Muro K, Oyama $\mathrm{T}$, Toh $\mathrm{Y}$, Udagawa $\mathrm{H}$, Uno $\mathrm{T}$, et al: Comprehensive registry of esophageal cancer in Japan 2010. Esophagus 14: 189-214, 2017.
2. Rudiger Siewert J, Feith M, Werner M and Stein HJ: Adenocarinoma of the esophagogastric junction: Results of surgical therapy based on anatomical/topographic classification in 1,002 consecutive patients. Ann Surg 232: 353-3361, 2000.

3. van Hagen P, Hulshof MC, van Lanschot JJ, Steyerberg EW, van Berge Henegouwen MI, Wijnhoven BP, Richel DJ, Nieuwenhuijzen GA, Hospers GA, Bonenkamp JJ, et al: Preoperative chemoradiotherapy for esophageal or junctional cancer. N Engl J Med 366: 2074-2084, 2012.

4. Lerut T, Coosemans W, Decker G, De Leyn P, Nafteux P and Van Raemdonck D: Anastomotic complications after esophagectomy. Dig Surg 19: 92-98, 2002.

5. Yoshida N, Yamamoto $H$, Baba $H$, Miyata $H$, Watanabe $M$, Toh Y, Matubara H, Kakeji Y and Seto Y: Can minimally invasive esophagectomy replace open esophagectomy for esophageal cancer? Latest analysis of 24,233 esophagectomies from the Japanese national clinical database. Ann Surg, 2019 [Epub ahead of print].

6. Dickinson KJ and Blackmon SH: Management of conduit necrosis following esophagectomy. Thorac Surg 25: 461-470, 2015.

7. Takemura M, Fujiwara Y, Moriura K, and Hori T: A case of residual esophageal necrosis after lower esophagectomy for early esophageal cancer. J Jap College Surgeons (Nihon Gekakei Rengo Gakkaishi) 36: 612-616, 2011.

8. Liebermann-Meffert DM, Meier R and Siewert JR: Vascular anatomy of the gastric tube used for esophageal reconstruction. Ann Thorac Surg 54: 1110-1115, 1992.

9. Urschel JD: Esophagogastrostomy anastomotic leaks complicating esophagectomy: A review. Am J Surg 169: 634-640, 1995.

10. Gurvits GE: Black esophagus: Acute esophageal necrosis syndrome. World J Gastroenterol 16: 3219-3225, 2010.

11. Gurvitis GE, Shapsis A, Lau N, Gualtieri N and Robilotti JG: Acute esophageal necrosis: A rare syndrome. J Gastroenterol 42: 29-38, 2007.

(i) $\odot$ This work is licensed under a Creative Commons Attribution-NonCommercial-NoDerivatives 4.0 International (CC BY-NC-ND 4.0) License. 